\title{
ELECTRON BEAM POSITION MONITOR (EBPM) DIAGNOSTICS FOR DIAMOND
}

\author{
M J Dufau, D M Dykes, R J Smith \\ CLRC Daresbury Laboratory, Warrington, UK
}

\begin{abstract}
This paper presents a global view of the proposed diagnostic systems for DIAMOND, but discusses in greatest detail the EBPMs, including data acquisition and control. Details of Total Current Monitor systems, and an active, beam position based interlock system for protecting ID vessels against thermal damage, by beam mis-steer, are also included.
\end{abstract}

\section{INTRODUCTION}

The DIAMOND light source project is proposed as the replacement for the Synchrotron Radiation Source (SRS) machine currently situated at Daresbury Laboratory. This accelerator source will consist of a main ring and most likely a gun, linac or microtron, and booster pre-injection system. These combined components will be capable of providing full energy injection and allow 'top up mode' running if required.

The Electron Beam Position Monitoring (EBPM) and ancillary diagnostic systems for DIAMOND will be extensive, comprehensive and complex. A fully instrumented pre-injector system is envisaged, complemented by high specification installed monitors in the main machine, to achieve the demanded performance.

Table 1: Machine Parameters

\begin{tabular}{|c|c|}
\hline Lattice & 20 Cell, DBA \\
\hline Energy [GeV] & 3.0 \\
\hline Accelerating Frequ. $(\mathrm{MHz})$ & 500 \\
\hline Circumference [m] & 396.8 \\
\hline Max length for IDs [m] & $\begin{array}{c}16 \times 4.76 ; \\
4 \times 8.14\end{array}$ \\
\hline Injection energy [GeV] & 3.0 \\
\hline Beam current $[\mathrm{mA}]$ & 300 \\
\hline Source size (fwhm) $\left[\mu \mathrm{m}^{2}\right]$ & $78 \times 14$ \\
Low beta straight; $\mathrm{h} \mathrm{x} \mathrm{v}$ & $335 \times 12$ \\
High beta straight; $\mathrm{h} \mathrm{x} \mathrm{v}$ & $331 \times 23$ \\
Long straight; $\mathrm{h} \times \mathrm{v}$ & $140(7$ per cell) \\
\hline Number of EBPMs & $1 \mu \mathrm{m}$ \\
\hline Accuracy $(\mathrm{H} \& \mathrm{~V})$ & $10 \%$ beam size \\
\hline Stability Required $(\mathrm{H} \& \mathrm{~V})$ & \\
\hline
\end{tabular}

All areas of beam transport, ramping and storage will be equipped with EBPMs and other diagnostic systems to provide position control, feedback and protection where necessary. Since the storage ring is the most critical with regards to operational stability and performance, it is possible to describe a reasonable installation scheme for the EBPMs associated with this, and other principal areas including the pre-injection stages. A review of the main storage ring parameters that directly concern the diagnostic systems are shown in Table 1.

\section{PRE-INJECTION DIAGNOSTICS}

\subsection{Beam Position Transport Diagnostics}

Beam extracted from the electron source will be produced with an RF structure at the machine frequency of $500 \mathrm{MHz}$. Bunched beam current pulses, after extraction at rates between 5 and $10 \mathrm{~Hz}$, will pass through an accelerating stage to the booster ring and then to the storage ring. The RF structure, relativistic nature and sufficiency of beam current allows the use of capacitive button type pickup EBPM heads to be located appropriately along the flight paths. For efficiency, it is hoped to rationalise the EBPM head to a single standard four button 'on axis' type giving two fully isolated measurements for each plane.

Since beam production is pulsed, the detection electronics will be of the single shot type. The processing detector electronics for such a system will be required to resolve beam position to the order of $200 \mu \mathrm{m}$. This can be achieved using synchronous homodyning detector systems[1] located in an adjacent accessible areas. Two pre-processing $180^{\circ}$ hybrids mounted local to the EBPM heads will be used to provide sum and difference outputs. These will be switched into the detector electronics to provide alternate readings from consecutive gun pulses of horizontal and vertical beam position. The output of these detectors will be fed into local analogue to digital conversion/digital signal processing (ADC/DSP) systems for quantisation then input into the control system to provide graphical mimic displays and steering feedback.

In keeping with the intended policy of integrating comprehensive diagnostics aids into all areas of DIAMOND, it is likely that some form of pulse-beam monitoring will be included in both transport lines. This will be designed around commercial devices, and will include an integrating beam charge monitor located after the gun to monitor gun efficiency. Fast AC current transformers may also be included at the ends of both transport lines to provide indications of pre-injector efficiency. 


\subsection{Booster Synchrotron Diagnostics}

Beam captured in the booster synchrotron will be accelerated to the storage ring energy at a frequency upto $10 \mathrm{~Hz}$. Four-button on-axis EBPM pickups will be installed at regular intervals around the circumference; position resolutions of $200 \mu \mathrm{m}$ should be adequate. As is the case for the beam transport system, the beam will be present only for a short time and so a single shot detector will also be suitable for application here. A high speed horizontal and vertical switching system and associated ADC/DSP system will allow readings to be taken from all EBPMs, in both planes, during the beam fill time, enabling feedback to be applied as required. Figure 1 shows a schematic arrangement of such a system. This is similar to that which would be fitted to the transport lines.

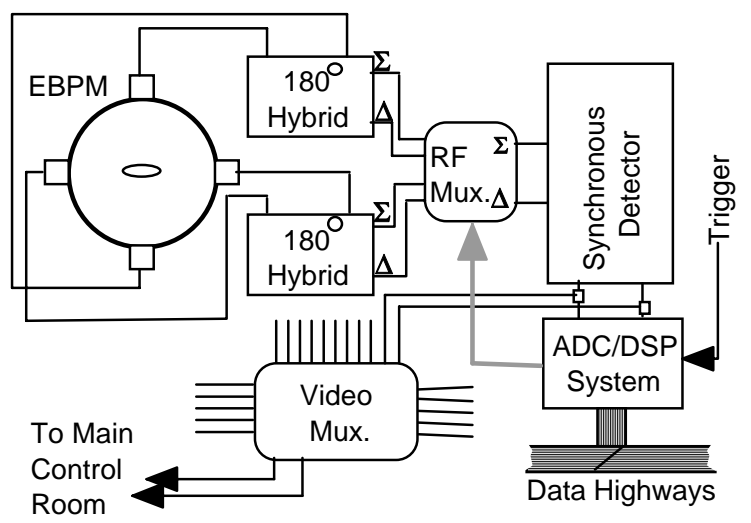

Figure 1: Basic Booster/Beam Transport EBPM System.

A delayed storage ring trigger pulse is used to arm the DSP for data collection. This will be used to gate out unwanted artefacts from the injection pulse.

Additionally a commercially available Total Current Monitor (or Parametric Current Transformers, PCT), plus, one on axis, quarter wavelength strip-line vessel will be installed within the booster. The strip-line will provide high sensitivity 'clean' signals direct to the Main Control Room (MCR). A suitable coaxial multiplexer will be used to provide processing to allow tune measurements to be made via a further synchronous detector and digital oscilloscope.

\section{STORAGE RING DIAGNOSTICS}

\subsection{Storage Ring Main EBPM System}

The storage ring main EBPM system will consist of 140 EBPMs, with at least 7 heads incorporated within each cell of the machine. Straights containing insertion devices will have heads that are fixed onto highly stable mounts to reduce mechanical movement, the greatest source of beam stability problems. These 'golden' EBPMs will share the same type of processing electronics as the remainder of the EBPMs, but with their improved mechanical stability, should provide the storage ring with known stable beam position alignment references. Again for efficiency, the pickup heads will be of a single rationalised design. A prototype design for the pickup head is shown in Figure 2.

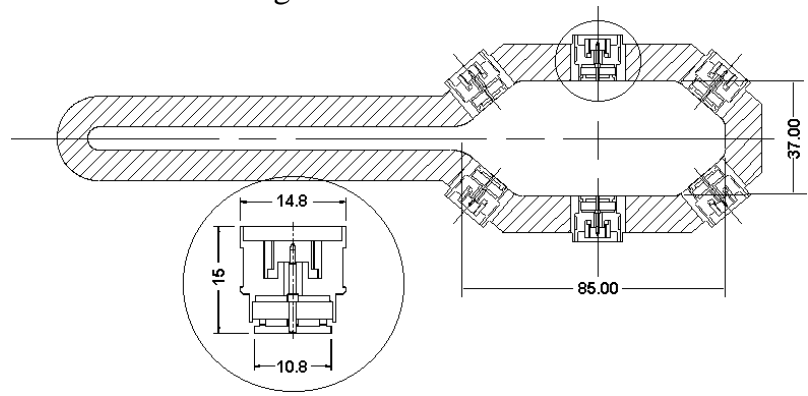

Figure 2: Typical Storage Ring EBPM Head vessel Design and capacitive button pickup.

Daresbury modified, ESRF type, capacitive button pickups are used for the EBPM head. These have reduced capacitance, which allows scanning wire bench calibration of the pickup head at low frequency $(5 \mathrm{MHz})$. Electrostatic finite element analysis of the EBPM head has been carried out in both planes using QuickField ${ }^{\mathrm{TM}}$. This has shown an adequate horizontal response but poor vertical response from the four buttons. This can be compensated for by the inclusion of separate on-axis buttons for the vertical plane, with the added advantage of completely de-coupling vertical beam movements from those in the horizontal plane. The electrical response for this configuration, shown in Figure 3 gives calibration factors near the centre of less than 12.5 for each plane.

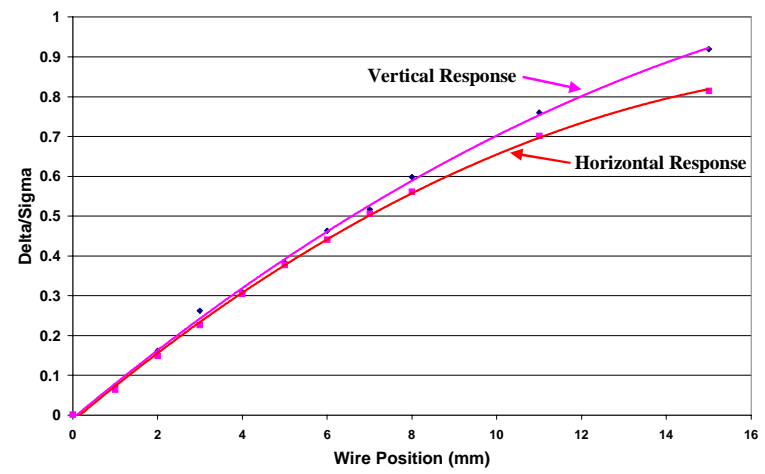

Figure 3: Horizontal and Vertical Calibration Curves.

Signal processing for these EBPMs will be done at several accessible marshalling points around the storage ring, using precision commercial detector electronics[2]. These detectors offer an improvement over the current Daresbury type detectors in that they have faster update rates (up to $2.5 \mathrm{kHz}$ ) and are more cost effective. The output of these detectors will pass into a DSP system for quantisation and presentation to the controls/positional feedback system. Once again, the same electronic systems will be applied to all EBPMs to standardise. The combination of the proposed small beam dimension and operating current specification of the detectors means that 
the system will heavily rely on mechanical stability to achieve its specification. A separate, dedicated single turn detector will be included on the 'golden' EBPM pickups to assist with machine operations and accelerator physics. Figure 4 below shows a typical detector system.

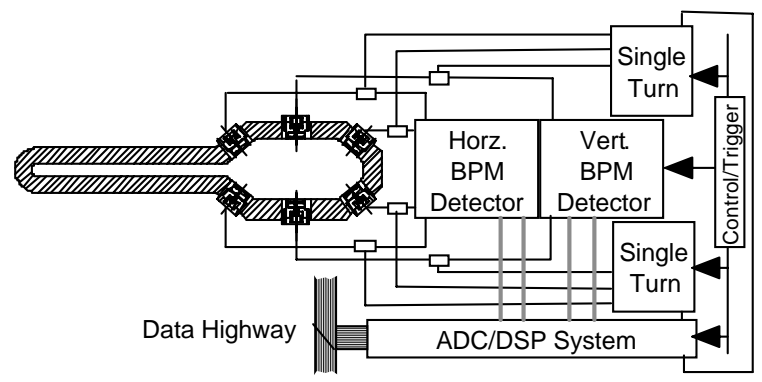

Figure 4: Typical Storage Ring 'Golden’ type EBPM

Additionally, a commercial PCT will be installed, along with an on axis diagnostic quarter wavelength strip vessel for basic beam diagnostics. A $45^{\circ}$ strip-line vessel will also be fitted to provide a beam tickler drive and pickup.

\subsection{Machine Protection and ID EBPMs}

For each of the IDs installed in the proposed source, an active electronic interlock system will be required, to protect the associated narrow-gap vessel from potential thermal damage, that would result from mis-steered beam. It is intended that the same successful philosophy of operation, as employed in both vessel protection systems currently operational in the SRS, will be applied to these future systems[3].

The two primary interlock inputs will be beam displacement information for the vessel, and temperature of the vessel walls and flanges, either of which will be capable of tripping off the machine RF at potentially damaging levels. As in the SRS, monitoring of the beam position will be designed around commercially available processing electronics operating from EBPMs within the ID vessel itself, while a thermocouple array interfaced via a Programmable Logic Controller, will monitor the temperature of the vessel walls. Because reliability is paramount, the integrity of all electronic hardware both primary and secondary, and also of that hardware providing support signals, will be monitored.

Since the application of this philosophy to the SRS vessel protection has led to a flexible design of satisfactory result, it is likely that the design will be applied en bloc to the requirements of DIAMOND. However local variations in the distribution of EBPMs may be required depending on specific vessel geometry.

During the period that the model for future vessel protection has been installed in the SRS, operational experience has highlighted the desirability for an integrated development enhancement offering diagnostic information regarding beam position, immediately prior to tripping the RF. Figure 5 shows a schematic, illustrating the proposal.

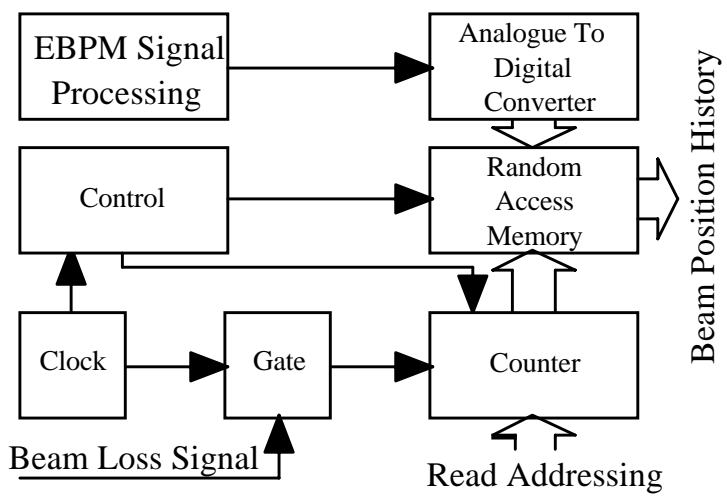

Figure 5: Beam Loss Data Recording System From Ids

Samples of the digitised beam position signal are stored in Random Access Memory (RAM) locations during read mode. A cyclic counter whose serial input is fed from a clock source provides the addressing for the locations. On beam loss the address counter is 'frozen' when the clock is inhibited, capturing in digital form the recent history of beam position. Addressing the locations via the parallel input of the counter can access the stored samples when the RAM is set to the write mode. Accessed samples could be read digitally or converted to analogue form.

\section{CONCLUSIONS}

This overview of proposed DIAMOND diagnostics presents realisable solutions for the major EBPM systems of the facility. At the present time, the systems discussed for the pre-injection stages would utilise old technology for the detectors, requiring extensive in-house development to produce an economically viable design of wide application. However, a suitable commercial unit is at present under development, and will probably be employed when available.

When active development of data processing systems commences, for economic reasons, it is intended that the DSP based data handling system, be of maximum versatility, with application to the full range of DIAMOND diagnostics.

\section{ACKNOWLEDGEMENTS}

Acknowledgements to M. Collier and C. Hill of the Daresbury Laboratory design office for providing engineering drawing.

\section{REFERENCES}

[1]T. Ring, "Beam Position Monitors For The High brightness Lattice", Internal Daresbury Lab. Technical Memorandum RING-85/134, (1985) [2]J. A. Hinkson, K. B. Unser., "Precision Analogue Signal Processor for Beam Position Measurements in Electron Storage Rings", Proceedings, $2^{\text {nd }}$ DIPAC, Travemunde, Germany, (May 1995)

[3]D. M. Dykes et al., "The SRS Multipole Wiggler Vacuum Vessel Protection System", Proceedings, $6^{\text {th }}$ EPAC, Stockholm, (1998) 